\title{
GENERALIZED $\beta$-EXPANSIONS, SUBSTITUTION TILINGS, AND LOCAL FINITENESS
}

\author{
NATALIE PRIEBE FRANK AND E. ARTHUR ROBINSON, JR.
}

\begin{abstract}
For a fairly general class of two-dimensional tiling substitutions, we prove that if the length expansion $\beta$ is a Pisot number, then the tilings defined by the substitution must be locally finite. We also give a simple example of a two-dimensional substitution on rectangular tiles, with a non-Pisot length expansion $\beta$, such that no tiling admitted by the substitution is locally finite. The proofs of both results are effectively one-dimensional and involve the idea of a certain type of generalized $\beta$-transformation.
\end{abstract}

\section{INTRODUCTION}

This paper was motivated by the study of simple tiling substitutions such as the substitution $S$ shown in Figure 1. To the left of the arrows, four prototiles
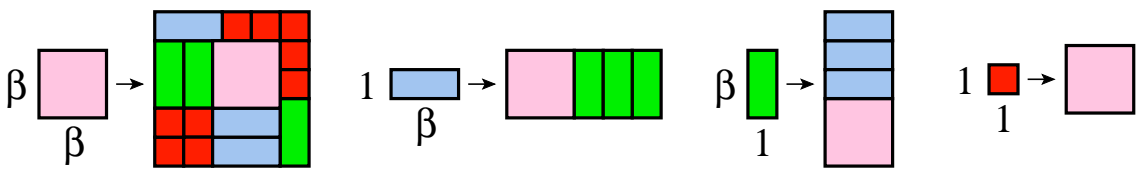

Figure 1. An inflate-and-subdivide rule $S$.

are shown along with their dimensions. The larger dimension, $\beta=(1+\sqrt{13}) / 2 \approx$ 2.3027756377 , is a root of the polynomial $p(x)=x^{2}-x-3$. The patches to the right of the arrows show the application of the substitution $S$ to each prototile. The substitution $S$ can be applied repeatedly, to produce arbitrarily large patches of tiles. The first few applications, starting with the large square prototile, are shown in Figure 2. Our interest in $S$ arises because its length expansion $\beta$ is not a Pisot number. An algebraic integer $\beta$ is called a Pisot number if $\beta>1$, and all its Galois conjugates $\beta^{\prime}$ satisfy $\left|\beta^{\prime}\right|<1$. Here, $\beta$ is not Pisot since its conjugate is $\beta^{\prime}=(1-\sqrt{13}) / 2 \approx-1.3027756377$.

It is known that whether or not the linear expansion of a tiling substitution $S$ is a Pisot number has implications for the tiling dynamical system that it defines. (We make the distinction between the linear expansion and the length expansion of a substitution tiling precise in Section 4.) Solomyak proved in [9] that a oneor two-dimensional self-similar tiling dynamical system with real linear expansion $\beta$ is weakly mixing if and only if $\beta$ is not Pisot. A similar result holds for a

Received by the editors June 6, 2005.

2000 Mathematics Subject Classification. Primary 52C20; Secondary 37B50.

Key words and phrases. Substitution sequence, self-similar tiling. 
two-dimensional self-similar tiling dynamical system with complex linear expansion $\lambda \in \mathbb{C} \backslash \mathbb{R}$. Solomyak showed that such a dynamical system is weakly mixing if and only if $\lambda$ is not a "complex Pisot" number. However, in the two-dimensional case the proofs of these results, as well as many similar results, use the additional assumption of "local finiteness" (a property that is automatic for the one-dimensional case). A tiling is defined to be locally finite ${ }^{1}$ if, up to rigid motions, it contains only finitely many two-tile configurations.

For substitution tilings, local finiteness follows from the "two-tile closure property" [6]. On the other hand, Danzer has conjectured [1] that non-locally finite tilings may in fact be generic for certain classes of substitutions. We will show that tilings admitted by the substitution in Figure 1 fail to be locally finite. It remains unclear the extent to which results like those in [9] can be extended to the non-locally finite case.

Although some examples of non-locally finite tilings have already appeared (see [1], see also [7]), our example has the benefit of simplicity. It belongs to a family [2] of tilings that can be created quite easily from any one-dimensional substitution. Moreover, our proof is based on a one-dimensional method that may be of independent interest.

To see how the lack of local finiteness appears from Figure 1, consider Figure 2, which shows three iterations of the large square tile. In the first iteration, we have circled a point where there is, in Danzer's terminology [1], a "misfit situation". Under the inflate-and-subdivide rule $S$ the tiles are inflated by the linear map

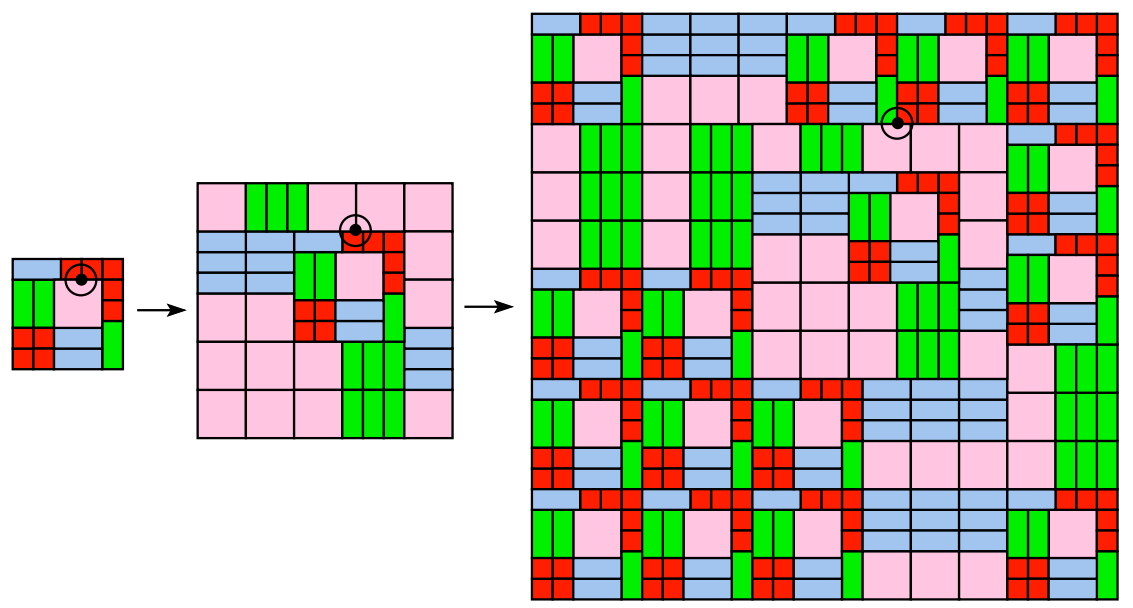

FIgURE 2. Three iterations of the larger square tile.

$\beta I$; we can follow the circled point's location under this linear map. Following the circled point through two more iterations, we see that the misfit it creates moves around relative to the tile below it. We will prove that this point occupies infinitely many locations with respect to the tile below it. Therefore the infinite tiling generated by this process is not locally finite.

\footnotetext{
${ }^{1}$ This property is also sometimes called "finite local complexity". In the theory Delone sets, however, "local finiteness" usually refers to a weaker property.
} 
Now one can begin to see why the arguments are one-dimensional. Inspection of Figure 1 shows that there are two kinds of upper edges: a long one and a short one, which we denote $l$ and $s$ respectively. The reader can verify that a long upper edge is substituted by a long followed by three short edges, $(l \rightarrow l s s s)$, and a short upper edge is substituted by a long one, $(s \rightarrow l)$. The circled point in Figure 2 is always on the corner of two of the tiles above it, but it is (we will show) always in the interior of an upper edge. Thus we restrict our attention to the one-dimensional substitution on the upper edges to prove our result. In fact, following the misfit through these iterates is analogous to applying a suitably defined "generalized $\beta$-transformation", which we discuss in the next section.

The organization of this paper is as follows. We first focus on the one-dimensional theory, which is itself of independent interest. In Section 2, we give the onedimensional definition of a substitution tiling and a generalized $\beta$-transformation, and we show how they are related. In Section 3 we give conditions that guarantee finite or infinite orbits of the generalized $\beta$-transformations and relate this result to one-dimensional tilings. In Section 4 we apply this machinery to tilings of $\mathbb{R}^{2}$.

\section{Definitions}

2.1. One-dimensional tiling substitutions. Let $\mathcal{A}=\{1, \ldots, m\}$ and let $\mathcal{A}^{*}$ denote the set of all (nonempty) finite words in $\mathcal{A}$. A substitution is mapping $\sigma: \mathcal{A} \rightarrow \mathcal{A}^{*}$. Given a substitution $\sigma$, the structure matrix is the $d \times d$ matrix $M$ with entries $M_{i, j}$ equal to the number of occurrences of the symbol $j$ in the word $\sigma(i)$. We require that $M$ has a real eigenvalue $\beta>1$ such that the right eigenvector $\vec{h}=\left(h_{1}, h_{2}, \ldots, h_{m}\right)$ has strictly positive entries. It is worth noting that PerronFrobenius theory ensures this in two special cases: irreducibility and primitivity. We say $M$ is irreducible if for each $i, j$ there is an $n$ for which $\left(M^{n}\right)_{i j}>0$. If a single $n$ works for all $i, j$ then we say $M$ is primitive. By the Perron-Frobenius theorem, if $M$ is irreducible, then there exists an $r>0$ for which $\vec{h} \in(r \mathbb{Q}(\beta))^{m}$.

Given a substitution $\sigma$, we construct a one-dimensional tiling substitution $S$ and a corresponding tiling $\tau$ of the line. The prototiles $\mathcal{P}=\left\{P_{1}, \ldots, P_{m}\right\}$ are intervals $P_{j}=\left[0, h_{j}\right)$ with lengths given by the entries of $\vec{h}$. A tile is a translation of a prototile $T=P_{j}+s=\left[s, s+h_{j}\right)$. A tiling patch $\pi$ is a finite sequence of tiles laid end to end:

$$
\pi=\left(P_{j_{1}}+s_{1}, P_{j_{2}}+s_{2}, \ldots, P_{j_{n}}+s_{n}\right)
$$

where

$$
s_{i+1}=s_{i}+h_{j_{i}} .
$$

Equivalently, in a tiling patch, the tiles are disjoint and the support, defined

$$
\operatorname{supp}(\pi):=\bigcup_{i=0}^{n} P_{j_{i}}+s_{i},
$$

is an interval. A tiling $\tau$ is an infinite set of disjoint tiles whose support is $\mathbb{R}$.

We call $s$ the left endpoint of the tiling patch $\pi$, and if $\operatorname{supp}(\pi)=[s, t)$, we call $t$ the right endpoint. We can specify a tiling patch $\pi$ by giving its left endpoint $s$ and the word $\mathbf{w}=j_{1} j_{2} \ldots j_{n}$ that specifies the prototiles that occur in its sequence. In particular, the patch in (1) is given by $\pi=\pi(s, \mathbf{w})$. 
Now we can define the tiling substitution $S$ corresponding to $\sigma$. It is the mapping from tilings to tiling patches defined by

$$
S\left(P_{j}+s\right)=\pi(\beta s, \sigma(j)) .
$$

Clearly we have $\operatorname{supp}(S(T))=\beta \operatorname{supp}(T)$, and $S(T+s)=S(T)+\beta s$. This allows us to extend $S$ to a mapping on tiling patches, and even to tilings. A tiling $\tau$ is said to be $S$-admissible if every patch $\pi \subset \tau$ is equivalent to a patch of $\pi^{\prime} \subset S^{n} P$ for some $P \in \mathcal{P}, n \in \mathbb{N}$. The set of all $S$-admissible tilings (which is always nonempty) is called a tiling space, and is written $X_{S}$.

Example 1. Consider the substitution $l \rightarrow l s s s, s \rightarrow l$ from the introduction. The structure matrix is

$$
M=\left[\begin{array}{ll}
1 & 3 \\
1 & 0
\end{array}\right]
$$

which has characteristic polynomial $p(x)=x^{2}-x-3$, and the Perron eigenvalue $\beta=(1+\sqrt{13}) / 2$. Taking $\vec{h}=(\beta, 1)$ as a positive right eigenvector, the prototiles are $P_{1}=[0, \beta)$ and $P_{2}=[0,1)$. These tiles have the same lengths as the edges of the tiles in Figure 1. We will continue our discussion of this example below.

2.2. Generalized $\beta$-transformations. Fix an interval $E=[0, B)$ and a real number $\beta>1$.

Definition 2.1. We say a mapping $F_{\beta}: E \rightarrow E$ is a generalized $\beta$-transformation if (i) $F_{\beta}$ is piecewise linear, (ii) $F_{\beta}$ is continuous from the right, and (iii) $F_{\beta}^{\prime}(x)=\beta$ for almost every $x \in I$.

More explicitly, $F_{\beta}$ is a generalized $\beta$-transformation if there is a partition of $E$ into $l$ subintervals $I_{j}=\left[x_{j-1}, x_{j}\right)$, with endpoints $0=x_{0}<x_{1}<\ldots<x_{l}=B$, and real numbers $y_{1}, y_{2}, \ldots, y_{l}$ with $\beta I_{j}-y_{j} \subseteq E$ for $j=1, \ldots, l$, so that

$$
F_{\beta}(x)=\beta x-y_{j} \text { whenever } x \in I_{j} .
$$

One example of this is the classical $\beta$-transformation $T_{\beta}:[0,1) \rightarrow[0,1)$, defined $T_{\beta}(x)=\beta x \bmod 1$ (see Renyi [5]). A type of generalized $\beta$-transformation, more general than what we consider here, was studied by Wilkinson [11]. He showed under several additional conditions that $F_{\beta}$ has an absolutely continuous invariant measure and is weakly Bernoulli with respect to the partition into the intervals $I_{j}$.

Definition 2.2. We call a generalized $\beta$-transformation $F_{\beta}$ algebraic if $\beta$ is an algebraic number and there is an $r \in \mathbb{R}$ for which

$$
x_{0}, \ldots, x_{l}, y_{1}, \ldots, y_{l} \in r \mathbb{Q}(\beta) .
$$

2.3. Substitution tilings and generalized $\beta$-transformations. Now let us think of the circled point in Figure 2 as being the origin situated in the interior of the one-dimensional tile that is the upper edge of a two-dimensional tile (Figure 3 ).

Suppose that $T=[s, t)$ is a tile with $s \leq 0$ and $t>0$, so that $0 \in T$. We call such a tile a central tile, illustrated in Figure 3 with $T=[1-\beta, 1)$. It follows that $0 \in \operatorname{supp}(S(T))=[\beta s, \beta t)$, and thus there is a unique central tile in $S(T)=$ $\left(P_{j_{1}}+s_{1}, P_{j_{2}}+s_{2}, \ldots, P_{j_{n}}+s_{n}\right)$, i.e., $0 \in P_{j_{i}}+s_{i}$ for some $i$. We can therefore define a mapping $\bar{S}$ from the set of all central tiles to itself by taking $\bar{S}(T)$ to be the central tile in $S(T)$. We are interested in the effects of iterating $\bar{S}$ on central tiles, but it is convenient to think of this process a little differently. 


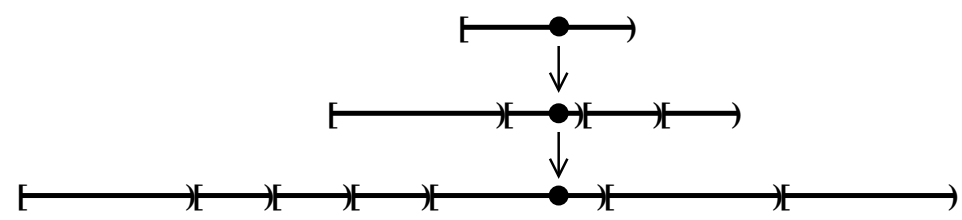

FigurE 3 . The map $\bar{S}$ finds the central tile after the substitution is applied.

First we consider the patch $\pi:=\pi(0,123 \ldots m)=\left(E_{1}, E_{2}, \ldots, E_{m}\right)$ where $E_{j}=$ $J_{j}+g_{j}$ with $g_{0}=0$ and $g_{j+1}=g_{j}+h_{j}$. We write the support of $\pi$ as $E=[0, B)$. The mapping $F: E \rightarrow E$ is defined as follows. For $x \in E$, find $j \in \mathcal{A}$ so that $x \in E_{j}$. Then $E_{j}-x$ is a central tile, and $\bar{S}\left(E_{j}-x\right)=E_{k}-y$ for some unique $k \in \mathcal{A}$ and $y \in \mathbb{R}$. We put $y=F(x)$, noting that $F(x) \in E_{k}$.

We may write $F$ explicitly as a generalized $\beta$-transformation by partitioning each $E_{j}$ into subintervals that will be sent by $F$ onto whole tiles (the canonical subdivision suggested by $S$ ). We obtain a partition $0=x_{0}<x_{1}<\ldots<x_{l}=B$ into intervals $I_{1}, \ldots, I_{l}$ such that for each $I_{k}$ there is a $j$ such that $F\left(I_{k}\right)=E_{j}$. The restriction of $F$ to $I_{k}$ is the linear map taking $I_{k}$ onto $E_{j}$. So for $x \in I_{k}$ we have

$$
F(x)=\beta\left(x-x_{k-1}\right)+g_{j-1} ;
$$

hence $y_{k}=-\beta x_{k-1}+g_{j-1}$.

Example 2. The generalized $\beta$-transformation for Example 1 is defined on the interval $E=[0,1+\beta)$ so that $E_{1}=[0, \beta)$ and $E_{2}=[\beta, \beta+1)$. The partition of $E$ given by the substitution is $x_{0}=0, x_{1}=1, x_{2}=1+1 / \beta, x_{3}=1+2 / \beta, x_{4}=$ $1+3 / \beta=\beta$, and $x_{5}=1+\beta$. Since $g_{0}=0$ and $g_{1}=\beta$, our translations are $y_{1}=0, y_{2}=0, y_{3}=1, y_{4}=2$, and $y_{5}=3+\beta$. The transformation is as shown in Figure 4 .

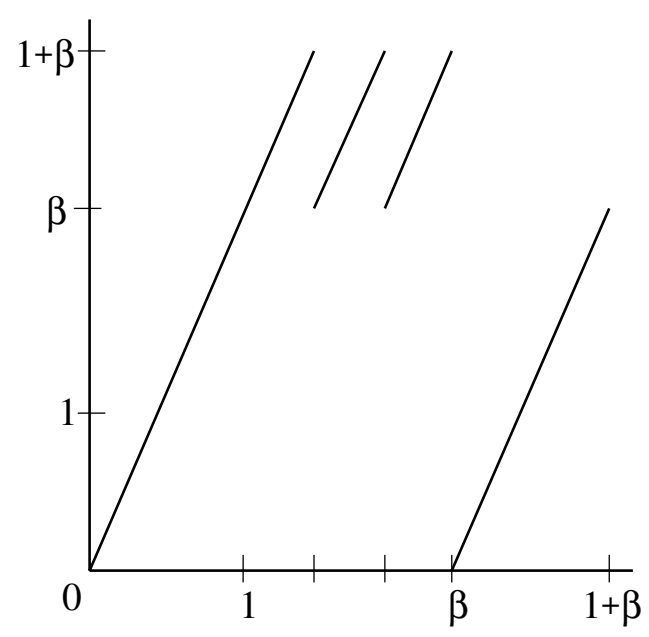

Figure 4. The map $F: E \rightarrow E$.

Since each interval in a tiling substitution expands by a factor of $\beta$, we have the following lemma. 
Lemma 2.3. The mapping $F$ given by a tiling substitution with expansion constant $\beta$ is an algebraic generalized $\beta$-transformation whenever $\vec{h} \in(r \mathbb{Q}(\beta))^{m}$.

This is certainly true whenever $M$ is irreducible or primitive.

\section{One-Dimensional RESUlts}

Suppose $\beta>1$ is a root of the irreducible monic polynomial $p(x)=g_{0}+g_{1} x+$ $g_{2} x^{2}+\ldots+x^{d} \in \mathbb{Z}[x]$, where $d>1$. We can identify $\mathbb{Q}(\beta)$ with $\mathbb{Q}^{d}$ via the basis $\left\{1, \beta, \beta^{2}, \ldots, \beta^{d-1}\right\}$ over $\mathbb{Q}$. Multiplication by $\beta$ in $\mathbb{Q}(\beta)$ corresponds to multiplication by $A$ in $\mathbb{Q}^{d}$, where

$$
A=\left[\begin{array}{cccc}
0 & 0 & \cdots & -g_{0} \\
1 & 0 & \cdots & -g_{1} \\
0 & 1 & \cdots & -g_{2} \\
\cdots & & & \\
0 & \cdots & 1 & -g_{d-1}
\end{array}\right]
$$

is the companion matrix of $p(x)$.

To fully understand the action of $A$, we embed $\mathbb{Q}^{d}$ in $\mathbb{C}^{d}$ (although if the eigenvalues are all real, it suffices to embed in $\mathbb{R}^{d}$ ). We define a surjective linear map $\phi: \mathbb{C}^{d} \rightarrow \mathbb{C}$ by

$$
\phi(\vec{w})=w_{0}+w_{1} \beta+w_{2} \beta^{2}+\ldots+w_{d-1} \beta^{d-1} .
$$

The restriction of $\phi$ to $\mathbb{Q}^{d}$ is the isomorphism between $\mathbb{Q}^{d}$ and $\mathbb{Q}(\beta)$. Moreover, for any $\vec{w} \in \mathbb{C}^{d}$,

$$
\phi(A \vec{w})=\beta \phi(\vec{w}) .
$$

The Galois conjugates $\beta_{2}, \beta_{3}, \ldots, \beta_{d}$ of $\beta$ are eigenvalues of $A$ with eigenvectors $\vec{v}_{2}, \vec{v}_{3}, \ldots, \vec{v}_{d}$. By linearity $\phi\left(A \vec{v}_{j}\right)=\phi\left(\beta_{j} \vec{v}_{j}\right)=\beta_{j} \phi\left(\vec{v}_{j}\right)$, but by (6) we also have $\phi\left(A \vec{v}_{j}\right)=\beta \phi\left(\vec{v}_{j}\right)$, and so $\phi\left(\vec{v}_{j}\right)=0$ for all $j=2,3, \ldots, d$. Let $V_{0}$ be the subspace of $\mathbb{C}^{d}$ spanned by $\vec{v}_{2}, \ldots, \vec{v}_{d}$, and let $V^{+}$be the (one-dimensional) eigenspace of $\beta$. Since $\phi$ is not the zero map, we can select the vector $\vec{v} \in V^{+}$with $\phi(\vec{v})=1$ as a basis vector.

Consider fixed an algebraic generalized $\beta$-transformation $F: E \rightarrow E$. Recalling the notation of Subsection 2.2 we have the partition of $E$ given by $0=x_{0}<x_{1}<$ $\ldots<x_{l}=B$ with $x_{j} \in \mathbb{Q}(\beta)$; we note that by definition $\beta\left(x_{j}-x_{j-1}\right) \leq B$. We denote the $j$ th subinterval as $I_{j}=\left[x_{j-1}, x_{j}\right)$, and we have $y_{1}, y_{2}, \ldots, y_{l} \in \mathbb{Q}(\beta)$ so that $\beta I_{j}-y_{j} \subseteq E$.

Since $\phi(\vec{u})=0$ for any $\vec{u} \in V_{0}$, we know that $\phi(c \vec{v}+\vec{u}) \in \mathbb{R}$ if and only if $c \in \mathbb{R}$. Thus we can define

$$
W_{[a, b)}=\{\vec{w}: \phi(\vec{w}) \in[a, b)\}=\left\{c \vec{v}+\vec{u}: \vec{u} \in V_{0} \text { and } c \in[a, b)\right\} \subseteq \mathbb{C}^{d}
$$

to be the set of all points in $\mathbb{C}^{d}$ that $\phi$ maps onto the real interval $[a, b)$.

Let $W_{E}$ denote the subset of $\mathbb{C}^{d}$ corresponding to $E$ and denote the subsets of $\mathbb{C}^{d}$ that correspond to the intervals $I_{j}$ as $W_{j}=W_{I_{j}}$; the $W_{j}$ s form a partition of $W_{E}$. There are unique vectors $\vec{w}_{1}, \vec{w}_{2}, \ldots, \vec{w}_{d}$ in $\mathbb{Q}^{d}$ for which $\phi\left(\vec{w}_{i}\right)=y_{i}$, and one can easily check that $A W_{j}-\vec{w}_{j} \subseteq W_{E}$. Thus we can define the map $f: W_{E} \rightarrow W_{E}$ as

$$
f(\vec{w})=A \vec{w}-\vec{w}_{j} \text { whenever } \vec{w} \in W_{j}
$$


and we see that

$$
\phi \circ f=F \circ \phi .
$$

Because our interest lies with the mapping $F$, the theorem below is stated in terms of it, but the result is actually proved for $f$ and then extended to $F$. The proof is mostly linear algebra and uses ideas similar to [1], [8] and [10] .

Theorem 3.1. Let $\beta, \beta_{2}, \ldots, \beta_{d}, E$, and $F$ be defined as above.

(1) If $\left|\beta_{j}\right|<1$ for all $j=2,3, \ldots, d$, then for any $x \in \mathbb{Q}(\beta) \cap E$ the orbit of $x$ under $F$ is finite and therefore eventually periodic.

(2) If $\left|\beta_{j}\right|>1$ for some $j \in\{2,3, \ldots, d\}$, then there exists an $x \in \mathbb{Q}(\beta) \cap E$ such that the orbit of $x$ under $F$ is infinite.

Proof. Since $\phi \circ f=F \circ \phi$ and the map $\phi$ is a bijection when restricted to $\mathbb{Q}^{d}$, it suffices to prove the results for $f$.

Suppose $\left|\beta_{j}\right|<1$ for all $j=2,3, \ldots, d$. Let $\vec{w} \in \mathbb{Q}^{d} \cap W_{E}$ and $\vec{w}_{i}$ from (7) be written in terms of the eigenvectors of $A$ as

$$
\vec{w}=c \vec{v}+\sum_{i=2}^{d} c_{i} \vec{v}_{i} \quad \text { and } \quad \vec{w}_{j}=w_{j, 1} \vec{v}+\sum_{i=2}^{d} w_{j, i} \vec{v}_{i} .
$$

For $i=2, \ldots, d$ choose

$$
r_{i}>\frac{\left|w_{j, i}\right|}{1-\left|\beta_{i}\right|} \quad \text { for all } \quad j \in 1,2, \ldots, d .
$$

Define the trapping region $\mathcal{R}_{\text {trap }}$ to be the set of all points in $W_{E}$ such that $c_{i} \leq r_{i}$.

We claim that $\mathcal{R}_{\text {trap }}$ is invariant under the action of $f$. Suppose $\vec{w} \in \mathcal{R}_{\text {trap }}$ with such a choice of values, and find $j \in 1,2, \ldots, l$ such that $\vec{w} \in W_{j}$. Then

$$
f(\vec{w})=A \vec{w}-\vec{w}_{j}=\left(\beta \vec{v}+\sum_{i=2}^{d} \beta_{i} c_{i} \vec{v}_{i}\right)-\left(w_{j, 1} \vec{v}+\sum_{i=2}^{d} w_{j, i} \vec{v}_{i}\right) .
$$

Since the component in the direction of $\vec{v}$ is unrelated to the definition of $\mathcal{R}_{\text {trap }}$, we see that we must have $\left|\beta_{i} c_{i}-w_{j, i}\right| \leq r_{i}$ for $i=2,3, \ldots, d$. This is true since $\left|w_{j, i}\right|<\left(1-\left|\beta_{i}\right|\right) r_{i}$ and $\left|c_{i}\right| \leq r_{i}$. This proves the invariance.

Now note that there is some $q \in \mathbb{Z}$ for which $\vec{w}$ and the $\vec{w}_{j}$ s are in $\frac{1}{q} \mathbb{Z}^{d}$. Since $A$ is an integer matrix, it leaves $\frac{1}{q} \mathbb{Z}^{d}$ invariant and so $f\left(\frac{1}{q} \mathbb{Z}^{d} \cap W_{E}\right) \subseteq \frac{1}{q} \mathbb{Z}^{d} \cap$ $W_{E}$. Choose the numbers $r_{i}$ for the trapping region so that $\vec{w} \in \mathcal{R}_{\text {trap }}$; clearly $f\left(\frac{1}{q} \mathbb{Z}^{d} \cap \mathcal{R}_{\text {trap }}\right) \subseteq \frac{1}{q} \mathbb{Z}^{d} \cap \mathcal{R}_{\text {trap }}$. Since $\frac{1}{q} \mathbb{Z}^{d} \cap \mathcal{R}_{\text {trap }}$ is a finite set, the orbit of $\vec{w}$ is finite and thus eventually periodic.

To prove the second part of the theorem we must show there is an infinite escape region $\mathcal{R}_{\text {esc }} \subset W_{E}$ for which orbits beginning in $\mathcal{R}_{\text {esc }}$ tend to infinity. Without loss of generality we may assume that $\left|\beta_{2}\right|>1$. Choose $r_{2}$ so that

$$
r_{2}>\frac{\left|w_{j, 2}\right|}{\left|\beta_{2}\right|-1} \quad \text { for all } \quad j=1,2, \ldots, d \text {. }
$$

We define

$$
\mathcal{R}_{e s c}=\left\{\vec{w}=c \vec{v}+\sum_{i=2}^{d} c_{i} \vec{v}_{i} \subset W_{E} \text { such that }\left|c_{2}\right|>r_{2}\right\}
$$


To prove the result, we must show that for any $\vec{w} \in \mathcal{R}_{\text {esc }} \cap \frac{1}{q} \mathbb{Z}^{d}$, the component of $f(\vec{w})$ in the direction of $\vec{v}_{2}$ exceeds that of $\vec{w}$ in modulus. Supposing again that $\vec{w} \in W_{j}$, by the computation in equation (9) this amounts to showing that $\left|\beta_{2} c_{2}-w_{j, 2}\right|>\left|c_{2}\right|$. By choice of $r_{2}$ we have that $\left|w_{j, 2}\right|<\left(\left|\beta_{2}\right|-1\right) r_{2} \leq\left(\left|\beta_{2}\right|-1\right)\left|c_{2}\right|$ and the result follows.

Corollary 3.2. Let $S$ be a one-dimensional tiling substitution with expansion constant $\beta>1$ and $\vec{h} \in(r \mathbb{Q}(\beta))^{m}$. Let $T$ be a central tile. (i) Suppose $\beta$ is a Pisot number. If the endpoints of $T$ belong to $\mathbb{Q}(\beta)$, then there exist $K, L \in \mathbb{N}$ so that $\bar{S}^{N L+K}(T)=\bar{S}^{K}(T)$ for all $N>0$. (ii) Suppose $\beta$ is non-Pisot. Then there is a $q \in \mathbb{N}$ and finite $E \subseteq \frac{1}{q} \mathbb{Z}[\beta]$ such that for any $s \in \frac{1}{q} \mathbb{Z}[\beta] \backslash E$, if $T=[-s, t)$ is central, then $\bar{S}^{K}(T) \neq \bar{S}^{L}(T)$ for any $K>L \geq 1$.

\section{TILING RESUltS}

In this section we study a class of self-similar substitution tilings in $\mathbb{R}^{2} \cong \mathbb{C}$. Our first result is that if a substitution $S$ has a Pisot length expansion, then the tilings it produces are locally finite. This solves a conjecture of Danzer [1] for the type of tilings that we consider. In this section we also prove that tilings generated by the substitution from Figure 1 are not locally finite.

We begin with some general definitions. A tile $T$ is a closed topological disk $D \subseteq \mathbb{C}$, together (possibly) with a label which is an element of some fixed finite set $\mathcal{A}$. We say $D=\operatorname{supp}(T)$ is the support of $T$. Two tiles are called equivalent if they carry the same label and have congruent supports.

We will fix a finite set $\mathcal{P}$ of inequivalent tiles, called prototiles, and assume all tiles $T$ are equivalent to tiles from $\mathcal{P}$. A packing is a collection of tiles that intersect only on their boundaries, and the support of a packing is the union of the supports of its tiles ${ }^{2}$. A tiling patch $\pi$ is a finite packing with a connected support. We denote the set of patches by $\mathcal{P}^{*}$. A tiling $\tau$ is a packing with support $\mathbb{C}$.

Let $\lambda \in \mathbb{C}$ with $|\lambda|>1$. Suppose $S: \mathcal{P} \rightarrow \mathcal{P}^{*}$ is such that for each $P \in \mathcal{P}$, the $\operatorname{supp}(S(P))$ is congruent ${ }^{3}$ to $\lambda \cdot \operatorname{supp}(P):=\lambda \cdot P$. We call $S$ a self-similar tiling substitution (or simply a substitution when there is no danger of confusion). We call $\lambda$ the linear expansion of $S$, equating multiplication by $\lambda$ with a linear map on $\mathbb{R}^{2}$; volumes must expand by a factor of $\lambda \bar{\lambda}$. We call $\beta=|\lambda|$ the length expansion of $S$ because it gives the expansion of one-dimensional objects.

The substitution $S$ extends in an obvious way to any tile $T$, and can thus be iterated to obtain infinitely large patches $S^{n}(P)$. Following the one-dimensional case, we say a tiling $\tau$ is $S$-admissible if for every patch $\pi \in \tau$ is equivalent to a patch $\pi^{\prime} \in S^{n}(P)$ for some $P \in \mathcal{P}$ and $n \geq 0$. We denote the set of $S$-admissible tilings $\tau$ by $X_{S}$.

The following lemma may be well-known, but we include a short proof for completeness.

Lemma 4.1. Let $\mathcal{P}$ be a finite prototile set and let $S$ be a tiling substitution on $\mathcal{P}$. No prototile in $\mathcal{P}$ contains a nontrivial circular arc in its boundary.

\footnotetext{
${ }^{2}$ In the one-dimensional case, it was convenient to take tiles as half-open and insist on disjointness. This also forced equivalence to preserve orientation, a restriction we do not impose here.

${ }^{3}$ In the event that there is more than one congruence between the supports $S(P)$ and $\lambda \cdot P$, we will use the implicit parameterization of the boundaries to ensure uniqueness of $S(T)$.
} 
Proof. Suppose the boundary of a prototile $P \in \mathcal{P}$ contains an arc of radius $r>0$ subtending an angle $\theta>0$. The boundary of $S^{n}(P)$ contains $\operatorname{arcs}$ of radius $\beta^{n} r$ subtending the angle $\theta$, and this implies that there are prototiles in $\mathcal{P}$ that have circular arcs of radius $\beta^{n} r$ in their boundaries. Since the prototile set is finite, we can find the maximum angle $\theta_{n}>0$ subtended by the $\operatorname{arc}$ of radius $\beta^{n} r$ in any prototile. The arclengths $\theta_{n} \beta^{n} r$ must go to zero in order for the prototiles to be topological disks.

Let $d_{\max }$ and $v_{\min }$ be the maximum diameter and minimum volume of prototiles in $\mathcal{P}$, respectively. The tiles inside of $S^{n}(P)$ adjacent to the circular arc of length $\theta \beta^{n} r$ lie inside a strip of volume

$$
\theta / 2\left[\left(\beta^{n} r\right)^{2}-\left(\beta^{n} r-d_{\max }\right)^{2}\right]+2 \pi d_{\max }{ }^{2},
$$

where $2 \pi d_{\max }{ }^{2}$ ensures the ends of the strip are covered. The maximum number of tiles that can fit inside this strip is

$$
\frac{\theta / 2\left[2 d_{\max } \beta^{n} r-d_{\max }^{2}\right]+2 \pi d_{\max }^{2}}{v_{\min }}<\frac{\theta d_{\max } \beta^{n} r}{v_{\min }}+K,
$$

where $K=(2 \pi-\theta / 2) d_{\max }{ }^{2} / v_{\min }$ does not depend on $n$. The minimum number of tiles required to cover the arc of length $\theta \beta^{n} r$ is $\left(\theta \beta^{n} r\right) /\left(\theta_{n} \beta^{n} r\right)=\theta / \theta_{n}$. For all $n=1,2, \ldots$ we must have

$$
\begin{aligned}
& \frac{\theta}{\theta_{n}} \leq \frac{\theta d_{\max } \beta^{n} r}{v_{\min }}+K, \text { or } \\
& \theta \leq \frac{\theta d_{\max }}{v_{\min }}\left(\theta_{n} \beta^{n} r\right)+K \theta_{n} .
\end{aligned}
$$

We have noted that both terms on the right must go to zero, forcing $\theta$ to be zero also. This contradiction completes the proof.

4.1. Local finiteness when the length expansion is Pisot. For the result we are going to prove, we will need some mild additional geometric hypotheses on prototiles and tilings. A straight edge $\ell$ in the boundary of a tile $T$ is a line segment that cannot be extended without leaving the boundary. Any such edge has a left and a right endpoint, relative to the boundary being traversed clockwise. The absence of circular arcs explains the terminology in the following definition.

Definition 4.2. A tile $T$ is called fractagonal if:

(1) $T$ has only finitely many straight edges, and

(2) for any straight edge $\ell$ of $T$, the rest of $T$ lies in the interior of a half-plane generated by $\ell$ (we call this weak convexity).

The authors conjecture that the first property is automatically satisfied by the prototiles of a self-similar tiling substitution, provided they are weakly convex.

We also need an assumption that for straight edges, collinearity implies comeasurability in the following sense:

Definition 4.3. We say a tiling substitution $S$ is a property- $(C)$ substitution if whenever two straight edges $\ell_{1}$ and $\ell_{2}$ are collinear in a tiling $\tau \in X_{S}$, there is an $r>0$ for which $\left|\ell_{1}\right|,\left|\ell_{2}\right| \in r \mathbb{Q}(\beta)$, where $\beta=|\lambda|$ is the length expansion of $S$.

Theorem 4.4. Suppose that $S$ is a property-(C) tiling substitution on a finite set $\mathcal{P}$ of fractagonal prototiles. If the length expansion of $S$ is a Pisot number, then any tiling in $\tau \in X_{S}$ is locally finite. 
Notes. (1) We point out two common assumptions that are not necessary here. The substitution need not be primitive in that the structure matrix $M$ may never satisfy $M^{n}>0$. Tilings in $X_{S}$ need not be translationally finite, i.e. prototiles may appear in an infinite number of orientations.

(2) The authors are not aware of any primitive tiling substitutions that are not property-(C).

(3) In his dissertation [3], Dirk Frettlöh obtained a similar result under much stronger assumptions, including that the substitution is primitive, the expansion is an integer, and that the tiles are polygonal.

Proof. A simple adjacency in $\tau \in X_{S}$ is a two-tile patch $\alpha \subset \tau$ where the intersection is along straight edges. Kenyon [4] proves that for any tiling $\tau$ made from a finite prototile set $\mathcal{P}$, if $\tau$ has an infinite number of inequivalent two-tile patches, then those patches are either simple adjacencies, or they occur along an entire circle of tile boundaries. By Lemma 4.1 the latter cannot occur in a substitution tiling. It follows that in order to show $\tau \in X_{S}$ is locally finite, it suffices to show that there are only finitely many simple adjacencies $\alpha$.

Fix a simple adjacency $\alpha$ in $\tau \in X_{S}$. Two straight edges define $\alpha$; the difference between two vertices, one selected from each edge, is called an offset of $\alpha$. Thus each $\alpha$ defines four different offsets. It suffices to show that the total number of offsets is finite.

Let the offset $\mathcal{O}$ of $\alpha$ be fixed. We want to see $\mathcal{O}$ as the difference of sums of edge lengths of tiles (see Figure 6). As such, it is convenient to consider edges as being intervals on the positive real axis. We call a tile $T$ placed below (resp. above) the real axis with a straight edge on the real axis a lower (resp. upper) tile. By applying a rigid motion, we can take edge representatives with their left (resp. right) endpoints at the origin. We call these edge sets $\mathcal{E}_{\ell}$ and $\mathcal{E}_{u}$.

By weak convexity, the substitution $S$ induces a one-dimensional substitution $S_{\ell}$ on $\mathcal{E}_{\ell}$ in the sense of Subsection 2.1. It is clear that the length expansion $\beta$ of $S$ is the expansion of $S_{\ell}$, so the one-dimensional substitution has a Pisot expansion constant. If $T$ is the lower tile containing $e \in \mathcal{E}_{\ell}$, we simply rotate $S(T)$ so that $\lambda \cdot e$ is mapped to $\beta \cdot e$ and look at the lower tiles of $\beta \cdot e$. Similarly we can create the one-dimensional substitution $S_{u}$ on $\mathcal{E}_{u}$ by considering the substitution on upper tiles. In particular, $S_{u}$ is just the reverse of $S_{\ell}$.

By property $(\mathrm{C})$, the edge sets $\mathcal{E}_{\ell}$ and $\mathcal{E}_{u}$ are partitioned into a finite number of subsets for which there are constants $r_{1}, \ldots, r_{k}>0$ with each $r_{i} / r_{j}, i \neq j$, irrational modulo $\beta$, such that each edge length is in $r_{i} \mathbb{Q}(\beta)$ for some $i$. Note that the application of $S_{\ell}$ or $S_{u}$ preserves these classes. Without loss of generality we may assume that the edges of the simple adjacency $\alpha$ have lengths in $\mathbb{Q}(\beta)$ (i.e. $r=1$ ).

By the construction of $\tau$, there is some smallest $n \geq 0$ for which there exists a tile $T$ such that the simple adjacency $\alpha$ is a sub-patch of $S^{n+1}(T)$. The intersection of the two tiles in $\alpha$ is contained in a line segment $L$ that is composed of tile edges and is maximal in the sense that it cannot be extended without either entering the interior of some tile in $S^{n+1}(T)$ or leaving $S^{n+1}(T)$ entirely. We call $L$ a fault line through the tile $S^{n+1}(T)$. Since $\mathcal{O}$ depends only on $\alpha$, we can use $L$ to compute the value of $\mathcal{O}$, even if the tiles surrounding $L$ in $S^{n+1}(T)$ do not appear in $\tau$ (except of course those in $\alpha$ ).

If $n=0$, then $S(T)$ can be moved to the positive $x$-axis so that an endpoint of $L$ lies at the origin. By the weak convexity assumption it follows that $L$ can be seen 
as a union of edges of upper tiles and also as a union of edges of lower tiles. In this case $L$, along with the information about which level-one tile it is in and which tile edges define it, is called an initial segment.

If $n>0$, then by the minimality of $n$, there must be two level- $n$ tiles whose boundaries intersect $\alpha$. From this we see that $L$ must contain a line segment $L^{\prime}$ that is a union of edges of level- $n$ tiles. Since the tiles are assumed to be fractagonal, weak convexity implies that if $S^{n+1}(T)$ is moved so that $L^{\prime}$ in on the $x$-axis, then $L^{\prime}$ is the union of edges of upper level- $n$ tiles and also the union of edges of lower level- $n$ tiles. Thus it is the image under $S^{n}$ of some initial segment $L^{\prime \prime}$.

Since there are only a finite number of level-one tiles up to equivalence, there are only a finite number of initial segments up to equivalence. Let $\mathcal{L}$ be a set of representatives of these equivalence classes, restricted to those that are composed of edges with lengths in $\mathbb{Q}(\beta)$, so that they are commensurate with the edges of $\alpha$. Without loss of generality we choose these representatives to be on the positive real axis with an endpoint at the origin. For each $L \in \mathcal{L}$, the endpoints of the lower tiles of $L$ create a partition $0=a_{0}<a_{1}<\ldots<a_{k_{\ell}}=|L|$, where $k_{\ell}$ is the number of lower tiles of $L$ and $|L|$ is the length of $L$. Any such $a_{i}$ for any initial segment $L$ will be called a prefix of $L$. Similarly, the endpoints of the upper tiles of $L$ create a partition $0=b_{0}<b_{1}<\ldots<b_{k_{u}}=|L|$ where $k_{u}$ is the number of upper tiles of $L$. A suffix of an initial segment $L$ is $|L|-b_{i}$, where $b_{i}$ is in the partition of $L$. In the same manner, we may compute prefixes of $S_{\ell}(e)$ for $e \in \mathcal{E}_{\ell}$ and suffixes of $S_{u}(e)$ for $e \in \mathcal{E}_{u}$, restricting again to edges with lengths in $\mathbb{Q}(\beta)$. Since $\left|S_{\ell}(e)\right|=\left|S_{u}(e)\right|=\beta|e|$, the prefixes will be partition elements of $[0, \beta|e|]$, and the suffixes will be $\beta|e|$ minus partition elements of $[0, \beta|e|]$. We collect all such prefixes into a prefix set $\mathcal{Q}_{p x}$ and such suffixes into a suffix set $\mathcal{Q}_{s x}$. It is important to note that both $\mathcal{Q}_{p x}$ and $\mathcal{Q}_{s x}$ are finite.

Since each $P \in \mathcal{P}$ is fractagonal, there are only a finite number of straight edges. Thus there is a $q \in \mathbb{N}$ so that all straight edge lengths in the class of the simple adjacency $\alpha$ are in $\frac{1}{q} \mathbb{Z}[\beta]$. This implies that the lengths of all initial segments, and the lengths of all prefixes and suffixes in $\mathcal{Q}_{p x}$ and $\mathcal{Q}_{s x}$ are also in $\frac{1}{q} \mathbb{Z}[\beta]$. Since offsets are the difference between a sum of upper edges and a sum of lower edges, $\mathcal{O} \subset \frac{1}{q} \mathbb{Z}[\beta]$.

We have already seen that $\mathcal{O}$ lies in a segment $L^{\prime}$ that is the image $\lambda^{n} L^{\prime \prime}$ for some initial segment $L^{\prime \prime}$. Let $L_{0}$ be the representative in $\mathcal{L}$ of $L^{\prime \prime}$. The tiles in $\alpha$, which intersect $L^{\prime}=\lambda^{n} \cdot L^{\prime \prime}$, are equivalent to lower and upper tiles $T_{\ell}$ and $T_{u}$ intersecting $\beta^{n} \cdot L_{0}$. In fact the edges of $T_{\ell}$ and $T_{u}$ are lower and upper edges that are edges in $S_{\ell}\left(L_{0}\right)$ and $S_{u}\left(L_{0}\right)$ when we consider $L_{0}$ in terms of its lower and upper tiles. The endpoints of edges in $\alpha$ used to compute $\mathcal{O}$ correspond to points $a \in T_{\ell}$ and $b \in T_{u}$ (see Figure 5), so to compute $\mathcal{O}$ we need to compute $|b-a|$. Without loss of generality assume $a \leq b$.

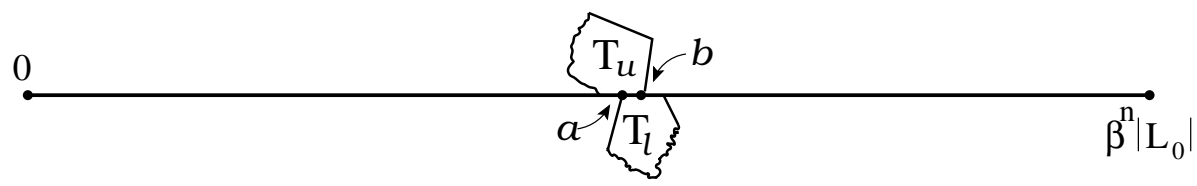

FiguRE 5. The simple adjacency $\alpha$ is equivalent to $T_{u} \cup T_{\ell}$. 
We may compute the left endpoint $a$ in terms of powers of $\beta$ applied to elements of $\mathcal{Q}_{p x}$; we do the same for the upper endpoint $b$ using powers of $\beta$ applied to elements of $\mathcal{Q}_{s x}$. (See Figure 6). Let $p_{n} \in \mathcal{Q}_{p x}$ be the largest prefix of $L_{0}$ for which $\beta^{n} p_{n} \leq a$. If equality holds, we have computed $a$, otherwise we continue. We denote by $e_{n-1}$ the lower edge immediately following $p_{n}$ in $L_{0}$. This edge will, after application of $S_{l}^{n}$, contain $a$ (otherwise $p_{n}$ wasn't maximal). So there is a largest prefix of $e_{n-1}$ which we call $p_{n-1} \in \mathcal{Q}_{p x}$ for which $\beta^{n} p_{n}+\beta^{n-1} p_{n-1} \leq a$. If equality holds, we are done and the remainder of the prefixes will be the zero prefix. If not, we have that $p_{n-1}$ is a prefix of $S_{\ell}\left(e_{n-1}\right)$ for some edge $e_{n-1} \in \mathcal{E}_{\ell}$, so the lower edge immediately following $p_{n-1}$ in $S_{\ell}\left(e_{n-1}\right)$ will, after application of $S^{n-1}$, contain $a$. We can continue in this fashion, choosing prefixes $p_{n-2}, \ldots, p_{0}$ until we obtain that $a=\sum_{k=0}^{n} \beta^{k} p_{k}$.

Similarly we can compute the value of $b$. Let $s_{n} \in \mathcal{Q}_{s x}$ be the largest suffix of $L_{0}$ for which $b \leq \beta^{n}\left(\left|L_{0}\right|-s_{n}\right)$. If equality holds, we are done and all subsequent suffixes are the zero suffix. If not we select the edge $e_{n-1}$ immediately preceding $\left|L_{0}\right|-s_{n}$ and find its suffix $s_{n-1}$ so that $b \leq \beta^{n}\left(\left|L_{0}\right|-s_{n}\right)-\beta^{n-1} s_{n-1}$. We continue choosing suffixes $s_{n-2}, \ldots, s_{0}$ until we obtain that $b=\beta^{n}\left|L_{0}\right|-\sum_{k=0}^{n} \beta^{k} s_{k}$.

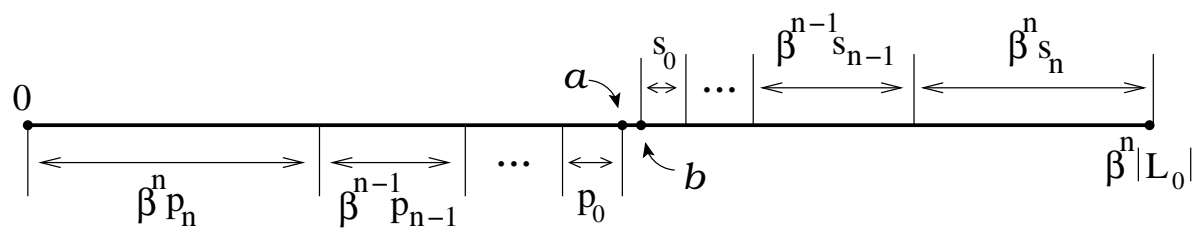

Figure 6 . The computation of $a$ and $b$ as seen in $\left[0, \beta^{n}\left|L_{0}\right|\right]$.

Thus the offset distance $\mathcal{O}$ is given by

$$
\mathcal{O}=\beta^{n}\left|L_{0}\right|+\sum_{k=0}^{n} \beta^{k}\left(-s_{k}-p_{k}\right) .
$$

Our interest, then, is in all possible combinations of suffixes and prefixes. We collect these into a difference set $\mathcal{D}=\left\{-s-p\right.$ such that $\left.s \in \mathcal{Q}_{s x}, p \in \mathcal{Q}_{p x}\right\}$ which we must show is finite.

If $\beta$ is an integer, then the result follows immediately since $\mathcal{O}$ is bounded by the length of the longest straight edge and is an element of $\frac{1}{q} \mathbb{Z}$.

If $\beta$ is not an integer, we need to use the ideas and notation of Section 3. Recall that the map $\phi: \mathbb{C}^{d} \rightarrow \mathbb{C}$ is a bijection between $\mathbb{Q}^{d}$ and $\mathbb{Q}(\beta)$, and that multiplication by the matrix $A$ in $\mathbb{Q}^{d}$ is equivalent to multiplication by $\beta$ in $\mathbb{Q}(\beta)$. We can find preimages $\vec{w}_{k} \in \mathbb{Q}^{d}$ such that $\phi\left(\vec{w}_{k}\right)=-s_{k}-p_{k}$ for $k=0, \ldots, n$. Since elements of $\mathcal{D}$ are in $\frac{1}{q} \mathbb{Z}[\beta]$, we have that each $\vec{w}_{k}$ must be in $\frac{1}{q} \mathbb{Z}^{d}$. Equation (10) becomes

$$
\mathcal{O}=\beta^{n}\left|L_{0}\right|+\sum_{k=0}^{n} \beta^{k} \phi\left(\vec{w}_{k}\right)=\beta^{n}\left|L_{0}\right|+\phi\left(\sum_{k=0}^{n} A^{k} \vec{w}_{k}\right) .
$$

We write the preimages of $\mathcal{D}$ restricted to $\mathbb{Q}^{d}$ in terms of the eigenvectors $\vec{v}_{1}, \ldots, \vec{v}_{d}$. Since $\phi$ projects onto $\vec{v}_{1}$ sending all other eigenvectors to 0 , and since $\mathcal{O}$ is necessarily less than the length of the longest edge, there is an $m_{1}>0$ that is an upper bound on the modulus of the component of $\phi^{-1}(\mathcal{O}) \cap \mathbb{Q}^{d}$ in the $\vec{v}_{1}$ direction. 
For any $j=2, \ldots, d$, denote by $m_{j}$ the maximum modulus of the component in the $\vec{v}_{j}$ direction of any preimage of the difference set $\mathcal{D}$ restricted to $\mathbb{Q}^{d}$. The modulus of the component of $\sum_{k=0}^{n} A^{k} \vec{w}_{k}$ in the $\vec{v}_{j}$ direction is less than or equal to

$$
\sum_{k=0}^{n}\left|\beta_{j}^{k}\right| m_{j}<\sum_{k=0}^{\infty}\left|\beta_{j}^{k}\right| m_{j}=\frac{m_{j}}{1-\left|\beta_{j}\right|},
$$

since $\left|\beta_{j}\right|<1$ by assumption when $j=2, \ldots, d$. So we see that the vectors which project onto $\mathcal{O}$ must lie in a bounded region. There are only a finite number of elements of $\frac{1}{q} \mathbb{Z}^{d}$ in a bounded region, so there are only a finite number of offsets $\mathcal{O}$, and hence only a finite number of simple adjacencies $A$. The tiling $x$ is locally finite.

Comment. A complex algebraic integer $\lambda$ is called a complex Pisot number if $|\lambda|>1$ and all algebraic conjugates $\lambda^{\prime} \neq \bar{\lambda}$ satisfy $\left|\lambda^{\prime}\right|<1$ (see [9], [6]). If $\lambda$ is complex Pisot, $\beta=|\lambda|$ may not be real Pisot (see [6] for an example). We do not know whether Theorem 4.4 always holds in the case of a complex Pisot linear expansion $\lambda$.

4.2. The Figure 1 example is not locally finite. Let $a$ denote the large square prototile from Figure 1. Since the substitution of any tile includes a tile of type $a$, it follows that a block of type $S^{n}(a)$ must appear in $S^{n+1}(e)$ for any tile type and for any $n=1,2, \ldots$ and so every tiling $x \in X$ must contain blocks of type $S^{n}(a)$ for all $n=1,2, \ldots$.

The circled point in Figure 2 appears inside the block $S(a)$. This point represents a vertex of the tiles above it and it intersects the upper boundary of the tile below it at a point $\beta-1$ units from the left endpoint of that boundary. Each time we apply the substitution, the circled point determines the offset between the tile below it and the two tiles above it. This offset is completely determined by $F$. If we can show that the orbit of $\beta-1$ is infinite, then this implies that there are an infinite number of offsets and hence the tiling is not locally finite. It is convenient to compute this orbit for $f$ rather than $F$.

Example 2 gives the algebraic generalized $\beta$-transformation associated with the substitution on upper edges of tiles. Noting that the eigenvalues are real, we compute the corresponding action $f$ in $W_{E} \subset \mathbb{R}^{2}$. The companion matrix of $\beta$ is

$$
A=\left[\begin{array}{ll}
0 & 1 \\
1 & 3
\end{array}\right] \text {. }
$$

We choose as the generator of $V^{+}$the vector $\vec{v}=\left(-\beta_{2}, 1\right)$ and as generator of $V_{0}$ we take $\vec{v}_{2}=(-\beta, 1)$. The vectors mapping onto $y_{1}, y_{2}, y_{3}, y_{4}$ and $y_{5}$ are given by $\vec{w}_{1}=(0,0), \vec{w}_{2}=(0,0), \vec{w}_{3}=(1,0), \vec{w}_{4}=(2,0)$, and $\vec{w}_{5}=(3,1)$. The sets $W_{j}$ have boundaries given by $V_{0}+\phi^{-1}\left(x_{j-1}\right)$ and $V_{0}+\phi^{-1}\left(x_{j}\right)$, where the inverse is taken in $\mathbb{Q}^{2}$. It is not difficult to check that the appropriate translation vectors are $(0,0),(1,0),(2 / 3,1 / 3),(1 / 3,2 / 3),(0,1)$ and $(1,1)$. (In this example one can also find these by looking at $A^{-1} \vec{w}_{j}$.) The regions $W_{j}$ are as shown in Figure 7 , along with the main eigenspace $V^{+}$.

We now establish an $f$-orbit which enters the escape region and thus tends to infinity. By our theorem, this orbit corresponds to an infinite $F$-orbit. We begin by computing the escape region. Looking in the proof of Theorem 3.1 (2), we see 


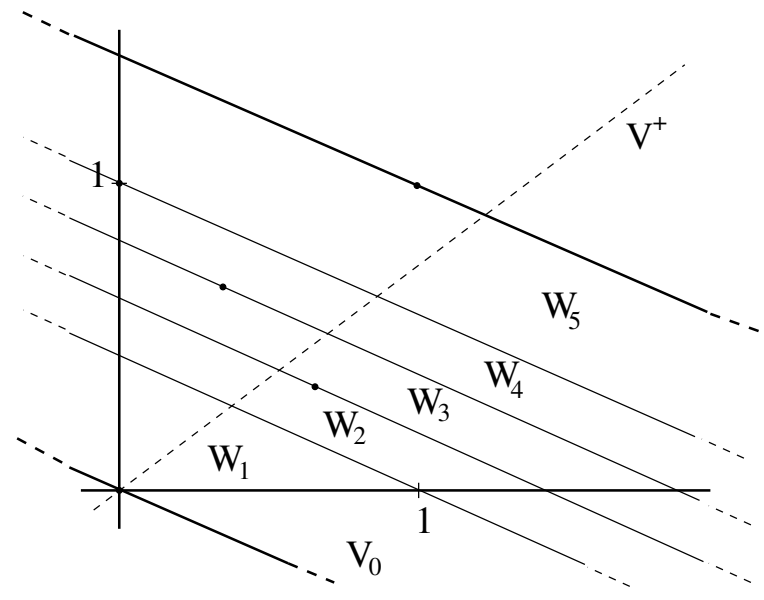

Figure 7. The regions $W_{j}$ in the domain $W_{E}$ of $f$.

that we must choose $r_{2}$ so that

$$
r_{2}>\frac{\left|w_{j, 2}\right|}{\left|\beta_{2}\right|-1} \quad \text { for all } \quad j=1,2,3,4
$$

We recall that $\vec{w}_{1}=(0,0), \vec{w}_{2}=(1,0), \vec{w}_{3}=(2,0)$, and $\vec{w}_{4}=(3,1)$ and compute their components in the $V_{0}$ direction:

$$
w_{1,2}=0, \quad w_{2,2}=\frac{-1}{\sqrt{13}}, \quad w_{3,2}=\frac{-2}{\sqrt{13}}, \quad w_{4,2}=\frac{-7+\sqrt{13}}{2 \sqrt{13}} .
$$

The largest of these in the modulus is $\left|w_{3,2}\right| \approx .5547$. We see that

$$
r_{2}>\frac{\left|w_{3,2}\right|}{\left|\beta_{2}\right|-1} \approx 1.8321
$$

so we simply choose $r_{2}=2$ to define $\mathcal{R}_{\text {esc }}$.

We will show that $\vec{w}=\phi^{-1}(\beta-1)=(-1,1)$ enters the escape region by computing the first several elements of its orbit. (This was made easier by using a cobwebbing program in Matlab to determine in which $W_{j}$ the point was at each step.) Now $(-1,1) \in W_{1}$, so $f(-1,1)=A(-1,1)-\vec{w}_{1}=(3,0)$. One can compute that $(3,0) \in W_{4}$, so $f(3,0)=A(3,0)-\vec{w}_{4}=(-3,2)$. Continuing in this fashion the next several iterates are $(5,-1),(-6,3),(9,-3),(-11,6),(15,-6)$. The orbit enters the escape region at $(-6,3)$ and the iterates begin to grow quite quickly. It is instructive and surprising to draw the region $W_{E}$, which is infinitely long and quite thin, and plot these points which must remain inside but which bounce further and further away from the origin.

\section{REFERENCES}

1. L. Danzer, Inflation species of planar tilings which are not of locally finite complexity, Proc. Steklov Inst. Math. 239 (2002), pp. 118-126. MR1975139 (2004c:52033)

2. N. P. Frank, Non-constant length $\mathbb{Z}^{d}$ substitutions, in preparation.

3. D. Frettlöh, Nichtperiodische Pflasterungen mit ganzzahligem Inflationsfaktor, Ph.D. dissertation, University of Dortmund, 2002.

4. R. Kenyon, Rigidity of planar tilings, Invent. Math. 107 (1992), 637-651. MR1150605 (92m:52049) 
5. A. Renyi, Representations for real numbers and their ergodic properties, Acta Math. Acad. Sci. Hungar 8 (1957), 477-493. MR0097374 (20:3843)

6. E. A. Robinson, Symbolic dynamics and tilings of $\mathbb{R}^{d}$, Proceedings of Symposia in Applied Mathematics 60 (2004), 81-119. MR2078847 (2005h:37036)

7. L. Sadun, Some generalizations of the pinwheel tiling, Discrete Comp. Geom. 20, no. 1 (1998), 79-110. MR1626703 (99e:52029)

8. K. Schmidt, On periodic expansions of Pisot numbers and Salem numbers, Bull. London Math. Soc. 12 (1980), 269-278. MR576976 (82c:12003)

9. B. Solomyak, Dynamics of self-similar tilings, Ergodic Theory Dynamical Systems 17 (1997), 695-738. Errata, Ergodic Theory Dynamical Systems 19 (1999), 1685. MR1452190 (98f:52030)

10. W. Thurston, Groups, Tilings, and Finite State Automata, AMS Colloquium Lecture Notes, American Mathematical Society, Boulder, 1989.

11. K. M. Wilkinson, Ergodic properties of a class of piecewise linear transformations, Z. Wahr. verw. Gebiete 31 (1975), pp. 303-328. MR0374390 (51:10590)

Department of Mathematics, Vassar College, Box 248, Poughkeepsie, New York 12604

E-mail address: nafrank@vassar.edu

Department of Mathematics, George Washington University, Washington, DC 20052

E-mail address: robinson@gwu.edu 